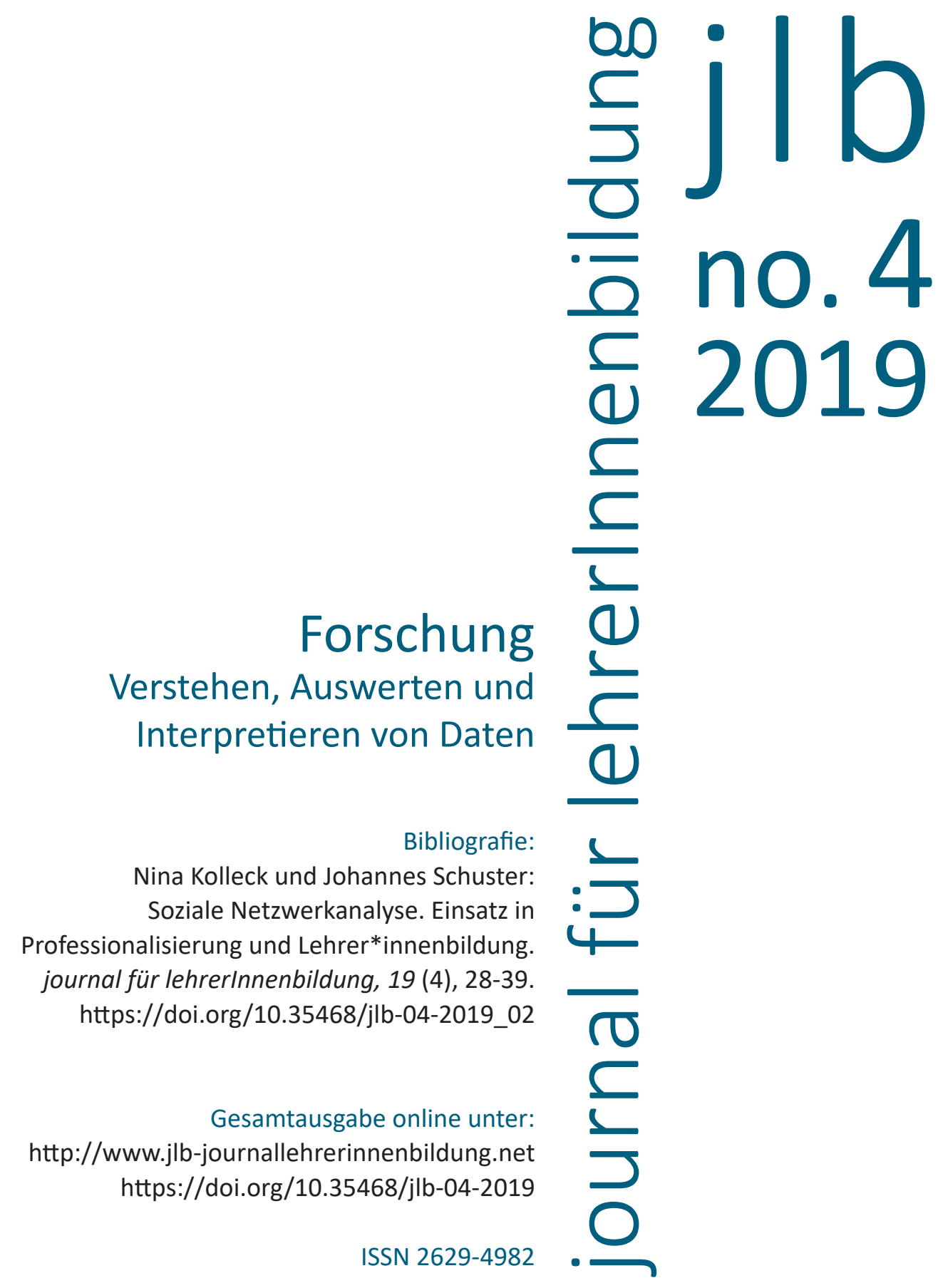


02 Nina Kolleck und
Johannes Schuster

\section{Soziale Netzwerkanalyse. Einsatz in Professionalisierung und Lehrer*innenbildung}




\section{Einleitung}

In den letzten Jahren sind soziale Netzwerke in der Schule zunehmend in den Fokus von Forschungsarbeiten gerückt (u.a Kolleck, 2016). In diversen Studien wurde gezeigt, dass für Prozesse der Lehrkräftebildung und Schulentwicklung nicht allein individuelles Handeln entscheidet, sondern soziale Beziehungen und Interaktionen einflussreich sind. So wirken Netzwerke bspw. innerhalb von Lehrer*innenkollegien auf die Motivation und Zufriedenheit der Lehrkräfte, aber auch innerhalb von Schulklassen auf die Motivation und Leistung der Schüler*innen. Die Erkenntnisse über die Beschaffenheit und die Strukturen schulischer Netzwerke, sei es innerhalb des Kollegiums oder in Klassenzimmern, können folglich dabei helfen, das verborgene Potenzial, das den Beziehungen zugrunde liegt, sowie Weiterentwicklungsmöglichkeiten zu identifizieren und Prozesse der Schulentwicklung und der Professionalisierung zu initiieren oder zu unterstützen.

Doch trotz der vielfältigen Einsatzmöglichkeiten und der möglichen Erkenntnisgewinne: Während sich seit einigen Jahren Wissenschaftler*innen zunehmend mit schulischen Netzwerken auseinandersetzen (Kappauf \& Kolleck, 2018) und auf Methoden der sozialen Netzwerkanalyse (SNA) zurückgreifen, zählt das Thema schulische Netzwerke noch nicht zum Bestandteil der Ausbildung von Lehrkräften. Eine Integration der SNA in die Lehrkräftebildung birgt jedoch viele Chancen. Unter anderem könnten hierdurch die Bedeutung sozialer Beziehungen und Beziehungsstrukturen vermittelt und ein Verständnis für die Gestaltung von Netzwerken sowie die Anwendungsmöglichkeiten netzwerkanalytischer Techniken im Kontext von Schulentwicklung und Professionalisierung erreicht werden. Der vorliegende Beitrag hat daher zwei Ziele. Erstens wird die Relevanz von sozialen Netzwerken in der Schule aufgezeigt. Zweitens wird ein Verfahren der Erhebung und Analyse sozialer Netzwerke im Schulkontext skizziert. Ziel ist es, den Einsatz dieses Verfahrens in Schulen exemplarisch darzustellen, sodass es in der Lehrer*innenbildung eingesetzt und zukünftigen Lehrkräften vermittelt werden kann. 


\section{Hintergrund und Forschungsstand}

Die Netzwerkanalyse ist seit einigen Jahrzehnten nicht mehr allein Gegenstand unterschiedlicher technischer und naturwissenschaftlicher Disziplinen, sondern hat Eingang in die Sozial- und Bildungswissenschaften gefunden. Mit dem Ziel, Muster und Eigenschaften sozialer Netzwerke zu identifizieren, die zwischen Akteur*innen, d. h. Individuen oder Organisationen (teils auch Objekten, Gegenständen oder Textbestandteilen), bestehen, werden mit Verfahren der Netzwerkanalyse unterschiedliche schulbezogene Fragestellungen adressiert. So werden u. a. Informationen über die Beziehungen oder Verbindungen zwischen den Akteur*innen, bspw. Lehrkräften oder Schüler*innen, erhoben. Diese Beziehungen können unterschiedliche Konzepte abbilden, z. B. Vertrauen, Kooperationen oder Sympathie (Freundschaft), aber auch Abneigung oder Mobbing. In der methodischen Literatur werden Netzwerke häufig als Einheiten definiert, die aus Akteur*innen (sogenannte Knoten) und den Beziehungen zwischen den Akteur*innen (Kanten) bestehen (Wassermann \& Faust, 1994). Netzwerke können gerichtet oder ungerichtet sein, wobei gerichtete Beziehungen einen Ursprung und ein Ziel haben (z. B. Sympathie-Beziehungen), während ungerichtete Netzwerke symmetrische Beziehungen, wie bspw. die gemeinsame Bearbeitung einer Aufgabe, abbilden.

Die soziale Eingebundenheit in der Klassengemeinschaft spielt eine wichtige Rolle für Schüler*innen. Dabei ist nicht nur die Anzahl an Beziehungen wichtig, sondern auch die strukturelle Lage im Gesamtnetzwerk (z. B. ob die eigenen Freund*innen viele andere Kontakte haben oder man selbst eher Teil einer ausgeschlossenen Clique ist) (Thomas, 2000). Studien zeigen, dass Peer-Beziehungen einen wichtigen Faktor bei der Entwicklung des Selbstkonzepts darstellen, da sich Schüler*innen dabei stark an anderen orientieren (Molloy, Gest \& Rulison, 2011). Darüber hinaus gibt es Hinweise, dass soziale Beziehungen in der Schule Auswirkungen auf Schulleistungen haben. In (nicht-repräsentativen) Studien wurde bspw. gezeigt, dass Schüler*innen dazu neigen, sich den Leistungen befreundeter Peers anzugleichen (Zander, Kolleck \& Hannover, 2014). Außerdem wirken sich Beziehungen zu Mitschüler*innen auf den individuellen Wissenserwerb aus (Wentzel, Russell \& Baker, 2014). Auch für Lehrkräfte weisen Studien auf einen positiven Zusammenhang von Netzwerken und Motivation hin. Sind 
Lehrkräfte gut in das Kollegium integriert bzw. Teil eines eng vernetzten Kollegiums, arbeiten sie motivierter und sind eher dazu bereit, zusätzliche Aufgaben zu übernehmen. Dies kann wiederum zu besseren Leistungen der Schüler*innen beitragen (Vangrieken, Dochy, Raes \& Kyndt, 2015).

Über Netzwerke innerhalb von Schulen hinaus bilden sich auch zwischen schulischen und außerschulischen Einrichtungen oder Personen Netzwerke, die positive Effekte auf den Bildungserfolg von Schüler*innen sowie Bildungsgerechtigkeit versprechen (Kolleck, 2015). Dabei zeigen sich Vertrauen, Partizipation und die Identifikation mit gemeinsamen Zielen als entscheidende Prädiktoren (Kolleck, Rieck \& Yemini, 2019). Soziale Beziehungen, die auf Vertrauen basieren, können unter Umständen wiederum positiv auf die Offenheit in Netzwerken gegenüber und Akzeptanz von Innovationen in Schulen wirken (Tschannen-Moran, 2001; Kappauf \& Kolleck, 2018).

\section{Methode}

Die Netzwerkanalyse liefert ein breites Repertoire an Methoden, mit deren Hilfe soziale Beziehungen und Netzwerke erhoben und ausgewertet werden können. Für den vorliegenden Beitrag stellen wir am Beispiel einer fiktiven Schulklasse - exemplarisch eine mögliche Methode vor, mit deren Hilfe interpersonale Netzwerke (bspw. von Seminargruppen oder Klassen) erhoben, visualisiert und analysiert werden können. Hierbei werden Beziehungen direkt erfragt und anschließend graphisch dargestellt. Je nachdem, welche Informationen in dem Netzwerk abgebildet werden sollen, können unterschiedliche Inhalte der sozialen Beziehungen ermittelt werden, z. B. Freundschaftsbeziehungen innerhalb der Klasse („Mit wem aus der Klasse bist du befreundet?“) oder Kooperationsbeziehungen („Mit wem aus der Klasse arbeitest du gerne zusammen?"). Der Fragebogen kann entweder alle Kinder aufführen, für die dann jeweils im Falle der Zustimmung ein Kreuz gesetzt wird, oder es kann nach den wichtigsten Personen gefragt werden („Nenne deine fünf besten Freunde“). Die zweite Variante ist sowohl für die Interviewten als auch für die spätere Auswertung stärker zu empfehlen und hat sich auch in wissenschaftlichen Studien behauptet (Merluzzi \& Burt, 2013). Sind zusätz- 
liche Informationen, die in das Netzwerk mit aufgenommen werden können (z. B. das Geschlecht, die Zugehörigkeit zu einem bestimmten Schwerpunkt, etc.), nicht ohnehin verfügbar, sollten diese bei der Erhebung der Daten miterfasst werden.

Für eine einfache und anschauliche Visualisierung von Netzwerken empfiehlt sich die kostenlos verfügbare Software Gephi. ${ }^{1}$ Diese bietet eine Vielzahl an Analyse- und Visualisierungstools, ohne dass Programmierkenntnisse notwendig sind (Schuster, Jörgens \& Kolleck, 2019). Im Folgenden beschreiben wir exemplarisch den Einsatz dieser Software in Schulen. In einem ersten Schritt müssen die Daten für die Visualisierung oder Analyse von Netzwerken erfasst werden. Zum einen können Daten manuell eingetragen werden. Dies erfolgt im Bereich „Datenlabor“. Hierzu müssen unter dem Reiter „Knoten“ zunächst alle Schüler*innen als Knoten hinzugefügt werden. Sind weitere Attribute der Schüler*innen bekannt (z. B. das Geschlecht oder die Zugehörigkeit zu einem bestimmten Schwerpunkt), können diese hier ebenfalls aufgenommen werden. Anschließend werden unter dem Reiter "Kanten“ die einzelnen Beziehungen als Kanten ausgewählt. Die Schülerin oder der Schüler, die oder der den Fragebogen ausgefüllt hat, ist in diesem Fall der "Ursprung“, die jeweils angegebenen Schüler*innen sind die „Ziele“. Alternativ können die Klassenliste (als „Nodes table") und die Liste der Beziehungen (als „Edges table“) auch als Excel-Dateien (möglich sind auch .csv- oder .txt-Formate) in Form einer Tabelle direkt in Gephi importiert werden. Beim Import solcher Listen ist Gephi jedoch etwas unflexibel und erfordert ein genaues Vorgehen. Damit ein "Nodes table" eingelesen werden kann, muss die Spalte mit den Personennamen mit "Id“ überschrieben werden. Die Namen weiterer Attribute können frei gewählt werden. Beim „Edges table" ist darauf zu achten, dass die Spalte mit den Ursprungspersonen die Überschrift "Source“ und die der Zielpersonen "Target" trägt und dass außerdem die Namen mit denen des "Nodes tables" identisch sind.

Sind alle Kanten eingegeben oder eingelesen, können einige Koeffizienten für die Schüler*innen berechnet werden. Hierzu muss in die „Übersicht" gewechselt werden, wo unter „Statistiken“ verschiedene

1 Gephi kann kostenlos heruntergeladen werden unter https://gephi.org/users/ download/ [24.10.2019]. Tiefergehende Informationen zur Software und ihrer Nutzung finden sich bei Bastian et al. (2009). 
Maße berechnet werden. Diese lassen sich anschließend im „Datenlabor" für alle Knoten einsehen. In erster Linie informativ sind der Eingangsgrad (wie oft ein*e Schüler*in nominiert wurde) und der Ausgangsgrad (wie viele Klassenkamerad*innen ein*e Schüler*in nominiert hat). ${ }^{2}$ Der Eingangsgrad kann einen Hinweis auf die Beliebtheit und soziale Eingebundenheit geben, während der Ausgangsgrad eher den Wunsch nach Eingebundenheit darstellt (Zander, Kreutzmann \& Hannover, 2017). Diese Ergebnisse lassen bereits einige Erkenntnisse über die jeweiligen Rollen der einzelnen Schüler*innen innerhalb der Klassengemeinschaft zu. So können wenig beliebte Schüler*innen ebenso identifiziert werden wie solche, die eher zurückhaltend sind bzw. wenig den Kontakt zu anderen suchen. Unter der Prämisse, dass eine soziale Eingebundenheit wichtig für die Zufriedenheit und die Motivation ist (Deci \& Ryan, 2000), können in diesem Fall Interventionen überlegt werden, z. B. hinsichtlich einer Neugestaltung der Sitzordnung.

Neben der Individualebene bietet Gephi Informationen über das gesamte Netzwerk, also die Klassenebene. Diese Informationen bieten einerseits die Möglichkeit, verschiedene Klassen miteinander zu vergleichen. Andererseits enthalten Ergebnisse zu einer Klasse wertvolle Informationen für Lehrkräfte, ohne dass ein Vergleich vorgenommen werden muss. Zunächst liefert bereits der erste Schritt der Visualisierung des Netzwerks eine verständliche Darstellung der Beziehungsstrukturen, die ein Ausgangspunkt für Entwicklungsmöglichkeiten der Lehrkräfte sein kann. Die Visualisierung erfolgt ebenfalls im Bereich „Übersicht“. Für eine gut interpretierbare Abbildung wird häufig das ForceAtlas2-Layout genutzt. Mit Hilfe einer solchen Netzwerkabbildung lassen sich kleinere Gruppen (sogenannte Cliquen), die von der Gesamtgemeinschaft isoliert sind, ebenso identifizieren wie völlig isolierte Schüler*innen (siehe Abb. 1). In unserem Beispiel zeigt sich etwa, dass Lara von der Klasse isoliert ist und es außerdem eine unabhängige Dreierclique aus Lucy, Anna und Ben gibt.

2 Dies ist nur unter der Voraussetzung sinnvoll, dass eine unbegrenzte Anzahl von Personen angegeben werden konnte. 


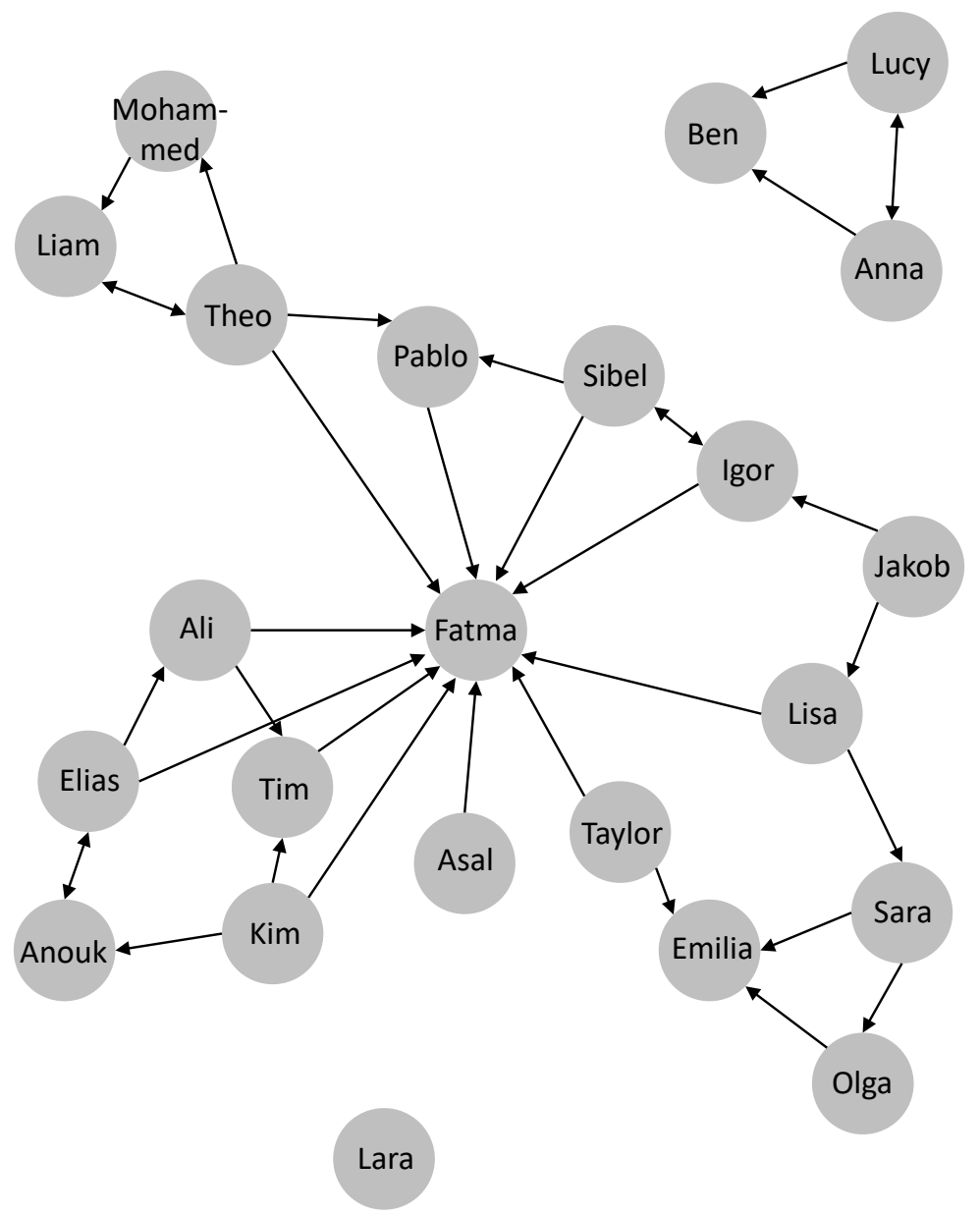

Abb. 1 Fiktives Klassennetzwerk (ohne Attribute)

Wurden weitere Attribute in die Übersicht aufgenommen, können diese anhand verschiedener Knotenfarben dargestellt werden (unter "Ausgestaltung" $\rightarrow$ "Partition“). Auf Grundlage der dadurch farbig gemachten Netzwerke lässt sich bspw. erkennen, ob es eine klare Geschlechtertrennung bei der Herstellung von Beziehungen gibt oder ob sich Gruppen je nach Zugehörigkeit zu einem bestimmten thematischen Schwerpunkt bilden. Auch ist es möglich, den (Eingangs- oder Ausgangs-)Grad anhand der Knotengröße darzustellen („Ausgestaltung" $\rightarrow$ "Ranking"). Durch die Visualisierung zusätzlicher Attribute zeigt sich somit für die Beispielklasse, dass Fatma sehr zentral ist. 
Außerdem scheint es eine Tendenz zu geben, gleichgeschlechtliche Freundschaften zu schließen (siehe Abb. 2).

Neben rein visuellen Informationen bietet Gephi auf der Klassenebene Maße, die für eine Beurteilung der Klassengemeinschaft genutzt werden können. Dazu zählt beispielsweise die Netzwerkdichte (findet sich ebenfalls unter "Statistiken“). Die Dichte bezieht sich auf den Anteil vorhandener Beziehungen im Verhältnis zu den insgesamt möglichen Beziehungen. Sie liefert daher eine wichtige Information darüber, wie eng die Klasse vernetzt ist. ${ }^{3}$

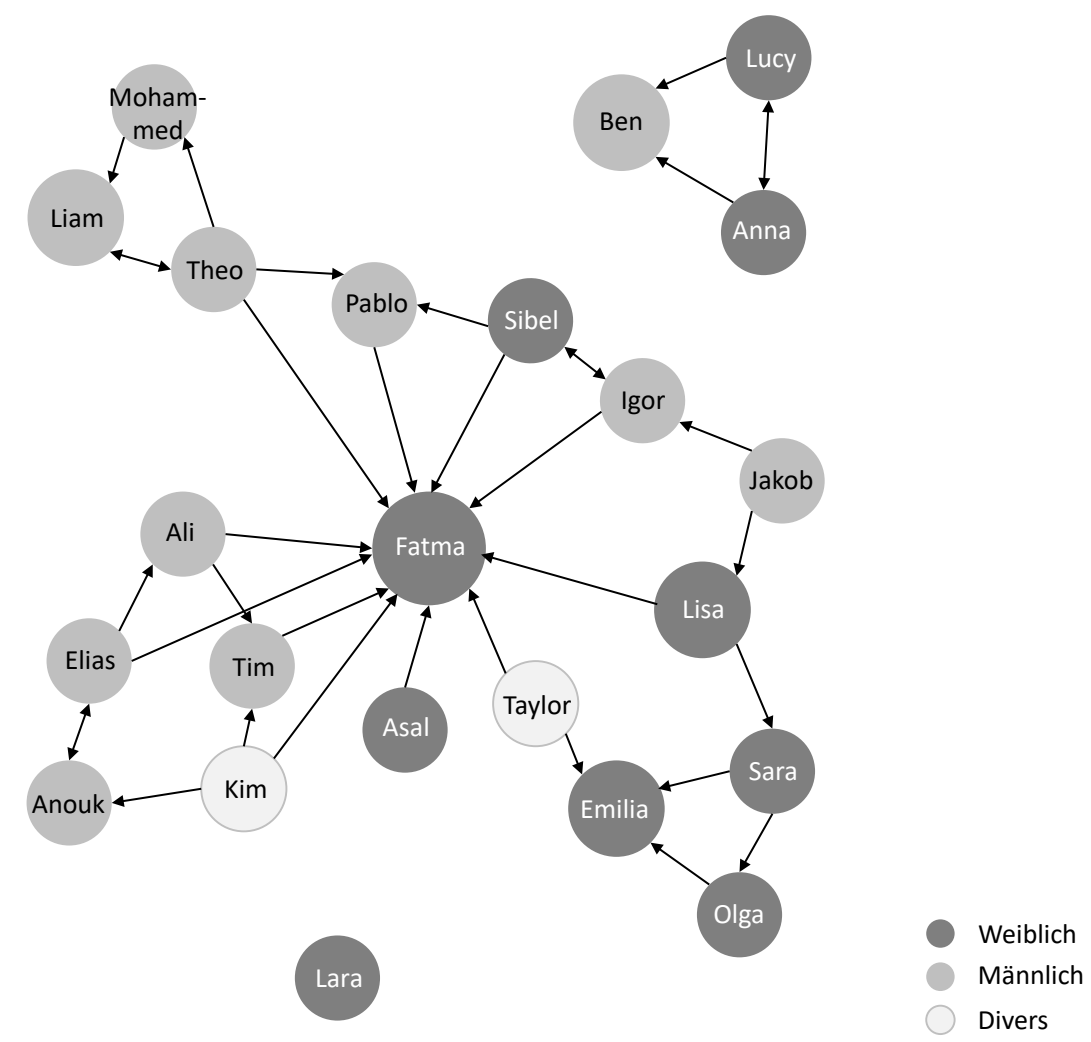

Abb. 2 Fiktives Klassennetzwerk (Graustufen = Geschlecht; Größe ist proportional zum Eingangsgrad)

3 Die Werte der Netzwerkdichte bewegen sich zwischen 0 und 1, wobei Werte näher an 0 eine schlechtere Vernetzung bedeuten; die Dichte lässt sich jedoch ohne andere Netzwerke vergleichbarer Größe und Art zumeist schwer interpretieren. 
Auf Grundlage dieser Erkenntnisse auf der Klassenebene lassen sich wiederum mögliche Konsequenzen für das Handeln der Lehrperson ableiten. Ist aus der Abbildung ersichtlich, dass sich die Klasse in viele kleine Gruppen aufteilt, könnten gemeinschaftsfördernde Maßnahmen zur Verbesserung der Klassengemeinschaft diskutiert werden. Für den Fall, dass sich diese Gruppen auf der Grundlage bestimmter Attribute ergeben, wäre es außerdem möglich, bei künftigen Gruppenarbeiten über eine gezielte Durchmischung nachzudenken. Deuten die Abbildungen darauf hin, dass die Klasse eigentlich sehr gut vernetzt ist und nur einzelne Schüler*innen aus der Gemeinschaft ausgeschlossen sind, könnten Einzelgespräche möglicherweise zu einer Verbesserung der Situation beitragen.

\section{Einsatz in der Lehrer*innenbildung}

Um das hier beschriebene Verfahren in der Lehrkräfteausbildung zu nutzen, ist es hilfreich, wenn die Seminarteilnehmenden zu Beginn der Einheit eigene Netzwerkdaten erheben - bspw. zu ihren individuellen Freundschaftsnetzwerken oder den Beziehungen in Schulklassen oder Kollegien. Diese können gegebenenfalls von den Teilnehmenden oder der Seminarleitung anonymisiert werden. Ist die Erhebung eigener Daten zu aufwendig, finden sich alternativ verschiedene Datensätze online, die für die Übung netzwerkanalytischer Methoden genutzt werden können. Dabei handelt es sich zumeist um vollständige Listen bestimmter Akteur*innen und ihrer Beziehungen zueinander. Um das Interesse der Studierenden zu erhöhen, bieten sich möglicherweise popkulturelle Netzwerke an, wie beispielsweise zu den Fernsehserien "Grey's Anatomy"4 oder "Game of Thrones". Diese Daten lassen sich ohne größeren Aufwand in Gephi nutzen, sodass die Studierenden die verschiedenen Möglichkeiten des Programms auch ohne eine aufwendige eigene Datenerhebung kennenlernen können.

Neben der hier beschriebenen Methode gibt es weitere Verfahren der Netzwerkanalyse, die in der Lehrer*innenbildung genutzt werden können. Die egozentrierte Netzwerkanalyse, bei der individuelle Netzwerke untersucht werden, bietet bspw. die Möglichkeit, detaillierte 
Einblicke in die Netzwerke einzelner Personen zu erhalten (Straus, 2010). Die Analyse schulischer Netzwerke birgt vielfältige Potenziale für Prozesse der Schulentwicklung, der Professionalisierung von Lehrkräften und der Verbesserung des Unterrichts. Zugleich sind Verfahren der Netzwerkanalyse anwendungsnah und aufgrund der Möglichkeiten, Ergebnisse übersichtlich und verständlich zu visualisieren, für Praxisakteur*innen oder Dozent*innen in der Lehrkräftebildung zugänglich. Das Wissen um die Anwendung und den Nutzen der sozialen Netzwerkanalyse im schulischen Kontext ermöglicht es Lehramtsstudierenden, die sozialen Strukturen innerhalb der Klasse oder des Kollegiums in ihrer Arbeit zu berücksichtigen. Auch aus diesen Gründen ist eine Integration verschiedener netzwerkanalytischer Methoden in die Lehrkräftebildung von hoher Relevanz und sollte zukünftig verstärkt berücksichtigt werden.

\section{Literatur}

Bastian, M., Heymann, S. \& Jacomy, M. (2009). Gephi: An Open Source Software for Exploring and Manipulating Networks. In Association for the Advancement of Artificial Intelligence (Ed.). Proceedings of the Third International Conference on Weblogs and Social Media. San Jose, California, May 17-20, 2009. Menlo Park, California: AAAI Press.

Deci, E. \& Ryan, R. M. (2000). The „What" and „Why“ of Goal Pursuits: Human Needs and the Self-Determination of Behavior. Psychological Inquiry, 11 (4), 227-268. https://doi.org/10.1207/S15327965PLI1104_01

Kappauf, Z. \& Kolleck, N. (2018). Vertrauen im Bildungsverbund: Skizze einer Theorie zu Dimensionen interpersonalen Vertrauens. Zeitschrift für Erziehungswissenschaft, 21 (5), 1045-1062. https://doi.org/10.1007/s11618-018-0812-4

Kolleck, N. (2015). Innovationen und Bildungslandschaften: Ergebnisse Sozialer Netzwerkanalysen. In R. Fischbach, N. Kolleck \& G. de Haan (Hrsg.). Auf dem Weg zu Nachhaltigen Bildungslandschaften. Lokale Netzwerke erforschen und gestalten (S. 55-68). Wiesbaden: VS/Springer Verlag. https://doi.org/10.1007/978-3-65806978-0_5

Kolleck, N. (2016). Uncovering influence through Social Network Analysis: the role of schools in Education for Sustainable Development. Journal of Education Policy, 23 (7), 308-330. https://doi.org/10.1080/02680939.2015.1119315

Kolleck, N., Rieck, A. \& Yemini, M. (2019). Goals aligned: Predictors of common goal identification in educational cross-sectoral collaboration initiatives. Educational Management Administration \& Leadership, 1-19. https://doi. org/10.1177/1741143219846906

Merluzzi, J. \& Burt, R. S. (2013). How many names are enough? Identifying network effects with the least set of listed contacts. Social Networks, 35 (3), 331-337. https://doi.org/10.1016/j.socnet.2013.03.004 
Molloy, L. E., Gest, S. D. \& Rulison, K. L. (2011). Peer Influences on Academic Motivation: Exploring Multiple Methods of Assessing Youths' Most "Influential“ Peer Relationships. The Journal of Early Adolescence, 31 (1), 13-40. https://doi. org $/ 10.1177 / 0272431610384487$

Schuster, J., Jörgens, H. \& Kolleck, N. (2019). Using Social Network Analysis to Study Twitter Data in the Field of International Agreements. London: SAGE Publications Ltd.

Straus, F. (2010). Netzwerkkarten - Netzwerke sichtbar machen. In C. Stegbauer \& R. Häußling (Hrsg.), Handbuch Netzwerkforschung (Bd. 3, Reihe Netzwerkforschung) (S. 527-538). Wiesbaden: VS Verlag für Sozialwissenschaften.

Thomas, S. L. (2000). Ties That Bind. The Journal of Higher Education, 71 (5), 591-615. https://doi.org/10.1080/00221546.2000.11778854

Vangrieken, K., Dochy, F., Raes, E. \& Kyndt, E. (2015). Teacher collaboration: A systematic review. Educational Research Review, 15, 17-40. https://doi.org/10.1016/j. edurev.2015.04.002

Wassermann, S. \& Faust, K. (1994). Social Network Analysis: Methods and Applications. Cambridge: Cambridge University Press.

Wentzel, K., Russell, S. \& Baker, S. (2014). Peer relationships and positive adjustment at school. In M. J. Furlong, R. Gilman \& E. S. Huebner (Eds.). Handbook of Positive Psychology in Schools (2nd ed.) (pp. 260-277). Hoboken: Taylor and Francis (Educational psychology handbook series).

Zander, L., Kolleck, N. \& Hannover, B. (2014). Editorial Note. Zeitschrift für Erziehungswissenschaft, 17 (5), 1-7. https://doi.org/10.1007/s11618-014-0545-y

Zander, L., Kreutzmann, M. \& Hannover, B. (2017). Peerbeziehungen im Klassenzimmer. Zeitschrift für Erziehungswissenschaft, 20 (3), 353-386. https://doi. org/10.1007/s11618-017-0768-9 
Nina Kolleck, Dr., Univ.-Prof. am Arbeitsbereich Politische Bildung der Universität Leipzig. Arbeitsschwerpunkte:

Netzwerke in Bildung und Erziehung, Heterogenität, Bildungspolitik und -verwaltung

nina.kolleck@uni-leipzig.de

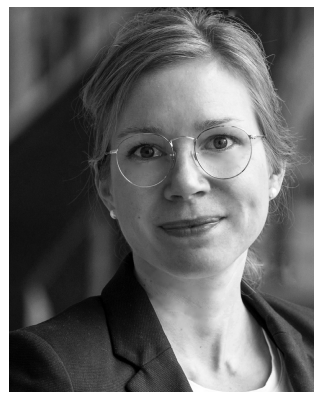

Johannes Schuster, wiss. Mitarbeiter am Institut für Bildungsforschung und soziale Systeme, Freie Universität Berlin. Arbeitsschwerpunkte: Implementierung von inklusiver Bildung, soziale Netzwerkanalyse

johannes.schuster@fu-berlin.de

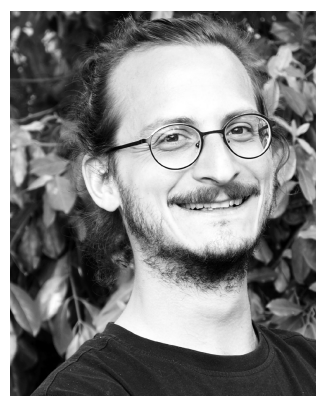

